\title{
NONEXPLOSION OF A CLASS OF SEMILINEAR EQUATIONS VIA BRANCHING PARTICLE REPRESENTATIONS
}

\author{
SANTANU CHAKRABORTY, ${ }^{*}$ University of Texas - Pan American \\ JOSE ALFREDO LÓPEZ-MIMBELA, ${ }^{* *}$ CIMAT
}

\begin{abstract}
We consider a branching particle system where an individual particle gives birth to a random number of offspring at the place where it dies. The probability distribution of the number of offspring is given by $p_{k}, k=2,3, \ldots$ The corresponding branching process is related to the semilinear partial differential equation $\partial u / \partial t=A u(t, x)+$ $\sum_{k=2}^{\infty} p_{k}(x) u^{k}(t, x)$ for $x \in \mathbb{R}^{d}$, where $A$ is the infinitesimal generator of a multiplicative semigroup and the $p_{k} \mathrm{~s}, k=2,3, \ldots$, are nonnegative functions such that $\sum_{k} p_{k}=1$. We obtain sufficient conditions for the existence of global positive solutions to semilinear equations of this form. Our results extend previous work by Nagasawa and Sirao (1969) and others.
\end{abstract}

Keywords: Branching particle system; semilinear partial differential equation; global solution

2000 Mathematics Subject Classification: Primary 60J80

Secondary $60 \mathrm{~J} 85$

\section{Introduction}

In this paper we investigate the existence of global solutions to the semilinear initial value problem

$$
\frac{\partial u}{\partial t}=A u(t, x)+F(t, x, u), \quad u(0, x)=f(x), x \in \mathbb{R}^{d} .
$$

Here $A$ is the infinitesimal generator of a linear contraction semigroup on the space $B\left(\mathbb{R}^{d}\right)$ of bounded measurable functions on $\mathbb{R}^{d}$, and

$$
F(t, x, u)=\sum_{k=2}^{\infty} p_{k}(x) u^{k}(t, x), \quad t \geq 0, x \in \mathbb{R}^{d},
$$

where the $p_{k}: \mathbb{R}^{d} \rightarrow[0,1]$ are measurable functions such that $\sum_{k=2}^{\infty} p_{k}(x)=1$.

In 1966 Fujita [1] studied finite-time blow up and existence of global solutions for the following semilinear equation:

$$
\frac{\partial u}{\partial t}=\Delta u(t, x)+u^{1+\alpha}, \quad u(0, x)=f(x), x \in \mathbb{R}^{d},
$$

where $\Delta$ is the Laplacian and $\alpha>0$. By means of analytic tools, he showed that if $\alpha>2 / d$ then the equation possesses both global and nonglobal solutions. This was considered to be

Received 19 September 2007; revision received 9 December 2007.

* Postal address: Department of Mathematics, University of Texas - Pan American, 1201 West University Drive,

Edinburg, TX 78539-2999, USA. Email address: schakraborty@utpa.edu

** Postal address: CIMAT, Apartado Postal 402, Guanajuato 36000, Mexico 
pioneering work at the time, inspiring both mathematicians and probabilists to come up with new and interesting results concerning global solutions and blow ups for semilinear equations. In fact, the authors of the first such work, Nagasawa and Sirao [8], mentioned in their introduction that they were motivated by Fujita's work.

Ikeda et al. [2], [3], [4], [5] gave a full description of branching Markov processes, which actually worked as a foundation for Nagasawa and Sirao to come up with the first probabilistic study of existence of global solutions and finite-time blow up for the equation

$$
\frac{\partial u}{\partial t}=A u(t, x)+c(x) u^{\beta}(t, x), \quad u(0, x)=f(x), x \in \mathbb{R}^{d},
$$

where $A$ is the infinitesimal generator of a linear nonnegative contraction semigroup on the space $B\left(\mathbb{R}^{d}\right)$ of bounded measurable functions on $\mathbb{R}^{d}, c(x)$ is a nonnegative bounded measurable function, and $\beta \geq 2$. They studied both the existence and nonexistence of global solutions of the above equation. Their method was based on probabilistic arguments relating to the branching Markov processes described in the work of Ikeda et al. They derived Fujita's result as a corollary of their result for the $\alpha$-Laplacian $A=-(-\Delta)^{\alpha / 2}, 0<\alpha \leq 2$. The space-time evolution of the branching population is as follows: an individual in the population develops a motion with generator $A$ and after an exponential lifetime, it is replaced by a population consisting of $\beta$ individuals at the site where it died.

Using a representation theorem in terms of branching particle systems, López-Mimbela [6] and López-Mimbela and Wakolbinger [7] extended the results of [8] to systems of semilinear equations with integral powers in their nonlinearities. Along the line of Nagasawa and Sirao's approach, López-Mimbela [6] showed that the solution $u_{t}(x)$ of the Nagasawa-Sirao equation (with initial condition $u_{0}=f$ ) has the representation

$$
u_{t}(x):=\mathrm{E}\left[\exp \left(S_{t}\right) \prod_{y \in X_{t}^{x}} f(y)\right]
$$

where $X_{t}^{x}$ is a branching particle system in $\mathbb{R}^{d}$ (with exponential individual lifetimes and offspring number $\beta$ ) starting from one ancestor at $x$ and $S_{t}$ is the total length of the family tree up to time $t$. This approach not only works for the $\alpha$-Laplacian but also for a wider class of generators.

In our case the offspring number is not fixed. It may vary from 0 to $\infty$. The motivation behind this is as follows. We remove the restriction that an individual in the population will give rise to a fixed number of offspring. Then instead of giving rise to $\beta$ offspring at any stage, we could have a probability distribution on the number of offspring. Such a probability distribution may be concentrated on finitely many values, say $\beta_{1}, \beta_{2}, \ldots, \beta_{p}$, etc. with coefficients $c_{1}(x), c_{2}(x), \ldots, c_{p}(x)$, etc. or infinitely many values, say all nonnegative integers $0,1,2, \ldots$ with probabilities, $p_{0}, p_{1}, p_{2}, \ldots$, say, adding up to 1 . In the former case the number of offspring is bounded, and in the latter case the number of offspring may be unbounded. Thus, in the former case we replace $c(x) u^{\beta}(t, x)$ by $\sum_{k=1}^{p} c_{k}(x) u^{\beta_{k}}(x)$, where $c_{k}(x)$ is bounded by a number, say $\left\|c_{k}\right\|$ for each $k$, and we consider

$$
\frac{\partial u}{\partial t}=A u+\sum_{k=1}^{p} c_{k}(x) u^{\beta_{k}}(t, x), \quad u(0, x)=f(x), x \in \mathbb{R}^{d} .
$$

In the latter case we replace $c(x) u^{\beta}(t, x)$ by some sort of probability generating function, namely $\sum_{k=0}^{\infty} p_{k}(x) u^{k}(t, x)$ with $p_{0} \equiv 0, p_{1} \equiv 0$, and $p_{k}(x) \equiv p_{k}$ for some constant $p_{k}$ for 
all $k$. So, we consider

$$
\frac{\partial u}{\partial t}=A u+\sum_{k=2}^{\infty} p_{k} u^{k}, \quad u(0, x)=f(x), x \in \mathbb{R}^{d} .
$$

In both (3) and (4) $A$ is the infinitesimal generator of a linear nonnegative contraction semigroup on the space $B\left(\mathbb{R}^{d}\right)$ of bounded measurable functions on $\mathbb{R}^{d}$. In the latter case the $p_{k} \mathrm{~s}$ are constants adding up to 1 .

By means of probabilistic representations of semilinear equations, here we shall study only the existence of global solutions of (1). When $A$ is the $d$-dimensional Laplacian and the number of offspring is fixed, we have a global solution provided that $f$ decays exponentially fast and $d$ is large enough. It is interesting to see if, in our case, similar conditions guarantee the existence of global positive solutions, namely, large mobility of individuals and quick decay of the initial value ensure boundedness of $u(t, x)$ for all $t \geq 0$. In the next section we introduce the ingredients we need to verify this, following Ikeda et al. [2], [3], [4], [5]. In Section 3 we describe Nagasawa-Sirao's condition for the existence of global solutions, and then we consider the bounded setup, i.e. the number of offspring is finite. We obtain conditions for global solutions which are similar to those of Nagasawa and Sirao. In Section 4 we consider the unbounded setup and observe that we need extra conditions for the existence of global solutions. In Section 5 we prove a preliminary result that precedes our main theorem for the unbounded case, and in Section 6 we prove several lemmas that are necessary to carry out our arguments in Section 5. In Section 7 we state and prove our main theorem, which actually gives conditions for the existence of global solutions in our case. We conclude with some interesting examples in Section 8.

\section{Preliminaries}

Before proceeding any further,following Ikeda et al. [2], [3], [4], [5], we need to introduce some background related to branching Markov processes. Nagasawa and Sirao [8] considered a branching Markov process where an individual in a particular generation gives birth to exactly $\beta$ offspring at the location of its death. Now we describe their setup.

Let $D$ denote a compact Hausdorff space with a countable open base, and let $B(D)$ denote the space of bounded Borel measurable functions on $D$. Here $B^{+}(D)$ denotes the set of nonnegative elements of $B(D)$. Let $\left\{T_{t} ; t \geq 0\right\}$ be a nonnegative contraction semigroup on $B(D)$ defined through a kernel $T_{t}(x, \mathrm{~d} y)$ such that the following four conditions are satisfied:

(i) $T_{t}(x, \cdot)$ is a nonnegative Borel measure on $D$ with $T_{t}(x, D) \leq 1$;

(ii) $T .(\cdot, B)$ is measurable on $[0, \infty) \times D$ for any Borel subset $B$ of $D$;

(iii) for any $t, s \geq 0, x \in D$, and Borel subset $B$,

$$
T_{t+s}(x, B)=\int T_{t}(x, \mathrm{~d} y) T_{t}(y, \mathrm{~d} B) ;
$$

(iv) for $f \in B(D)$,

$$
T_{t} f(x)=\int T_{t}(x, \mathrm{~d} y) f(y)
$$


We shall deal with positive mild solutions $v(t, x)$ to (1) with initial data $f \in B^{+}(D)$, which in the finite case is given by

$$
v(t, x)=T_{t} f(x)+\int_{0}^{t} \mathrm{~d} s T_{s}\left(\sum_{k=1}^{p} c_{k} v(t-s, \cdot)^{\beta_{k}}\right)(x)
$$

and in the infinite case is given by

$$
v(t, x)=T_{t} f(x)+\int_{0}^{t} \mathrm{~d} s T_{s}\left(\sum_{2}^{\infty} p_{k} v(t-s, \cdot)^{k}\right)(x) .
$$

We can apply the successive approximation method to obtain a (local) positive solution to (5). However, instead we shall use a linear dilatation of this equation, which is more appropriate for our purpose. We consider a linear integral equation on an enlarged space given by $S=\bigcup_{n=1}^{\infty} D^{n}$, where $D^{n}$ is a symmetric $n$-fold product of $D, n \geq 1$; see [2], [3], [4], and [5] for a detailed definition of $S$.

For $f \in B^{+}(D)$, Ikeda et al. [2], [3], [4], [5] set $\hat{f}=\prod_{j=1}^{n} f\left(x_{j}\right)$ when $x=\left(x_{1}, x_{2}, \ldots\right.$, $\left.x_{n}\right) \in D^{n} ; \hat{f}$ is then a measurable function on $S$ and $\hat{f} \in B^{+}(S)$ if $f \leq 1$.

Now we state some fundamental facts which will play an important role in our work; see [2], [3], [4], [5], and [8].

(i) There exist unique nonnegative kernels $T_{t}(x, \mathrm{~d} y)$ and $\Psi(x, \mathrm{~d} s \mathrm{~d} y)$ defined on $[0, \infty) \times S \times S$ and $S \times[0, \infty) \times S$, respectively, such that when $x=\left(x_{1}, x_{2}, \ldots, x_{n}\right) \in D^{n}$,

$$
\int_{S} T_{t}(x, \mathrm{~d} y) \hat{f}(y)=\prod_{j=1}^{n} T_{t} f\left(x_{j}\right),
$$

and in the finite case

$$
\begin{aligned}
\int \Psi(x, \mathrm{~d} s \mathrm{~d} y) \hat{f}(s, y) & =\mathrm{d} s \sum_{k=1}^{n} T_{s}\left(\sum_{j=1}^{p} c_{j} f(s, \cdot)^{k}\right)\left(x_{k}\right) \prod_{i \neq k, i=1}^{n} T_{s}(f(s, \cdot))\left(x_{i}\right) \\
& \leq \sum_{j=1}^{p}\left\|c_{j}\right\| \mathrm{d} s \sum_{k=1}^{n} T_{s}\left(f(s, \cdot)^{k}\right)\left(x_{k}\right) \prod_{i \neq k, i=1}^{n} T_{S}(f(s, \cdot))\left(x_{i}\right) \\
& =\sum_{j=1}^{p}\left\|c_{j}\right\| \int_{S^{n+j-1}} \Psi_{j}(x, \mathrm{~d} s \mathrm{~d} y) \hat{f}(y),
\end{aligned}
$$

and in the infinite case

$$
\begin{aligned}
\int \Psi(x, \mathrm{~d} s \mathrm{~d} y) \hat{f}(s, y) & =\mathrm{d} s \sum_{k=1}^{n} T_{s}\left(\sum_{j=2}^{\infty} p_{j} f(s, \cdot)^{k}\right)\left(x_{k}\right) \prod_{i \neq k, i=1}^{n} T_{s}(f(s, \cdot))\left(x_{i}\right) \\
& =\sum_{j=2}^{\infty} p_{j} \mathrm{~d} s \sum_{k=1}^{n} T_{s}\left(f(s, \cdot)^{k}\right)\left(x_{k}\right) \prod_{i \neq k, i=1}^{n} T_{s}(f(s, \cdot))\left(x_{i}\right) \\
& =\sum_{j=2}^{\infty} p_{j} \int_{S^{n+j-1}} \Psi_{j}(x, \mathrm{~d} s \mathrm{~d} y) \hat{f}(y),
\end{aligned}
$$


where $\sum_{2}^{\infty} p_{k}=1$. It can be seen that $\Psi_{j}(x, \mathrm{~d} s \mathrm{~d} y)$ is defined as follows:

$$
\int_{S^{n+j-1}} \Psi_{j}(x, \mathrm{~d} s \mathrm{~d} y) \hat{f}(y)=\sum_{k=1}^{n} T_{s}\left(f(s, \cdot)^{k}\right)\left(x_{k}\right) \prod_{i \neq k, i=1}^{n} T_{s}(f(s, \cdot))\left(x_{i}\right) .
$$

Moreover, if $x \in D^{n}$, the support of $T_{t}(x, \cdot)$ is concentrated on $D^{n}$ and the support of $\Psi_{j}(x, \mathrm{~d} s \cdot)$ is concentrated on $D^{n+j-1}$ for $j=1, \ldots, p$ in the former case and $j=2,3, \ldots$ in the latter case. Therefore,

$$
\int \Psi(x, \mathrm{~d} s \mathrm{~d} y) \hat{f}(s, y)=\sum_{j=2}^{\infty} p_{j} \int_{S^{n+j-1}} \Psi_{j}(x, \mathrm{~d} s \mathrm{~d} y) \hat{f}(y) .
$$

Having done this, we obtain the linear integral equation which is a linear dilatation of (1) with initial data $f$, given by

$$
u(t, x)=\widehat{T_{t} f(x)}+\int_{0}^{t} \int_{S} \Psi(x, \mathrm{~d} s \mathrm{~d} y) u(t-s, y), \quad x \in S, f \in B^{+}(S),
$$

where

$$
\widehat{T_{t} f(x)}=\int_{S} T_{t}(x, \mathrm{~d} y) \hat{f}(y)
$$

Then, we set

$$
\begin{gathered}
u_{0}(t, x)=\widehat{T_{t} f(x)}, \\
u_{k}(t, x)=\int_{0}^{t} \int_{S} \Psi(x, \mathrm{~d} s \mathrm{~d} y) u_{k-1}(t-s, y), \quad k \geq 1 .
\end{gathered}
$$

(ii) The function $u_{k}(t, x)$ is well defined and $\sum_{k=0}^{\infty} u_{k}(t, x)$ converges for sufficiently small $t>0$. Setting

$$
u(t, x)=\sum_{k=0}^{\infty} u_{k}(t, x)
$$

when the right-hand side converges, we conclude, as in [2], [3], [4], and [5], that $u$ is the minimal (local) solution of (5).

(iii) The most important property is considered to be the branching property:

$$
u(t, x)=\prod_{j=1}^{n} u\left(t, x_{j}\right) \quad \text { when } x=\left(x_{1}, \ldots, x_{n}\right) \in D^{n},
$$

which is also due to Ikeda et al. [2], [3], [4], [5].

\section{Conditions for the existence of global positive solutions in the bounded case}

As mentioned earlier, Nagasawa and Sirao [8] considered global solutions and blow ups for the following equation:

$$
\frac{\partial u}{\partial t}=A u(t, x)+c(x) u^{\beta}(t, x), \quad u(0, x)=f(x),
$$


which is (2) above. Here $A$ is the infinitesimal generator of a linear nonnegative contraction semigroup on the space $B\left(\mathbb{R}^{d}\right)$ of bounded measurable functions on $\mathbb{R}^{d}, c(x)$ is a nonnegative bounded measurable function, and $\beta \geq 2$. Then the conditions for global solutions are given by the following theorem.

Theorem 1. For $f \in B^{+}(D)$ satisfying

$$
(\beta-1)\|c\| \int_{0}^{\infty} \sup _{y \in D}\left(T_{t} f(y)\right)^{\beta-1} \mathrm{~d} t<1,
$$

there exists a global solution $u(t, x)$ of (2). Moreover, there exists a constant $M>0$ such that

$$
u(t, x) \leq M \widehat{T_{t} f(x)}
$$

The offspring number is $\beta$ in Nagasawa and Sirao's paper. In our case the offspring number is not fixed, it varies from $\beta_{1}$ to $\beta_{p}$ in the former case and from 2 to $\infty$ in the latter case. As mentioned earlier, in our setup, we replace $c(x) u^{\beta}(t, x)$ by either

$$
\sum_{k=1}^{p} c_{k}(x) u^{\beta_{k}}(x) \quad \text { or } \quad \sum_{k=2}^{\infty} p_{k}(x) u^{k}(t, x) .
$$

Hence, our semilinear equation is given by (3) or (4) accordingly. In this section we consider the former case when the number of offspring is bounded:

$$
\frac{\partial u}{\partial t}=A u(t, x)+\sum_{k=1}^{p} c_{k}(x) u^{\beta_{k}}(t, x), \quad u(0, x)=f(x) .
$$

Then, following the approach adopted in Nagasawa-Sirao's work, we can prove the following proposition.

Proposition 1. For any $t \geq 0$ and $x=\left(x_{1}, \ldots, x_{n}\right)$, we have

$$
u_{k}(t, x) \leq \frac{\prod_{i=0}^{k-1}\left(n+i\left(\beta_{\max }-1\right)\right)}{k !}\|c\|^{k} p^{k}\left(\int_{0}^{t} \sigma_{s}^{\beta_{\min }-1} \mathrm{~d} s\right)^{k} \widehat{T_{t} f(x)}
$$

where $\sigma_{s}=\sup _{s} T_{s} f, \beta_{\min }=\min \left(\beta_{1}, \ldots, \beta_{p}\right), \beta_{\max }=\max \left(\beta_{1}, \ldots, \beta_{p}\right)$, and $\|c\|=$ $\max \left(\left\|c_{1}\right\|, \ldots,\left\|c_{p}\right\|\right)$.

Proof. We have

$$
\begin{aligned}
u_{1}(t, x) & \leq\|c\| n\left(\sum_{j=1}^{p} \int_{0}^{t} \sigma_{s}^{\beta_{j}-1} \mathrm{~d} s\right) \widehat{T_{t} f(x)} \\
& \leq\|c\| n p\left(\int_{0}^{t} \sigma_{s}{ }^{\beta_{\min }-1} \mathrm{~d} s\right) \widehat{T_{t} f(x)} .
\end{aligned}
$$


Then,

$$
\begin{aligned}
u_{2}(t, x) \leq & \int_{0}^{t} \sum_{j=1}^{p} c_{j}(x) \int_{S^{n+\beta_{j}-1}} \Psi_{j}(x, \mathrm{~d} s \mathrm{~d} y) u_{1}(t-s, y) \\
\leq & \int_{0}^{t} \mathrm{~d} s \sum_{j=1}^{p}\|c\| \int_{0}^{t-s}\|c\| p\left(n+\beta_{j}-1\right) \sigma_{r}^{\beta_{\min }-1} \mathrm{~d} r \\
& \times \sum_{i=1}^{n} T_{s}\left(T_{t-s} f\left(x_{i}\right)^{\beta_{j}}\right) \prod_{l=1, l \neq i} T_{s}\left(T_{t-s} f\right)\left(x_{l}\right) \\
\leq & \int_{0}^{t} \mathrm{~d} s\|c\|^{2}\left(\sum_{j=1}^{p}\left(\int_{0}^{s} p\left(n+\beta_{j}-1\right) \sigma_{r}^{\beta_{\min }-1} \mathrm{~d} r\right) \sigma_{s}^{\beta_{j}-1}\right) \widehat{T_{t} f(x)} \\
\leq & \int_{0}^{t} \mathrm{~d} s\|c\|^{2}\left(\sum_{j=1}^{p} \int_{0}^{s} p\left(n+\beta_{j}-1\right) \sigma_{r}^{\beta_{\min }-1} \mathrm{~d} r\right) \sigma_{s}^{\beta_{\min }-1} \widehat{T_{t} f(x)} \\
\leq & \frac{n\left(n+\beta_{\max }-1\right)}{2} p^{2}\|c\|^{2}\left(\int_{0}^{t} \sigma_{s}^{\beta_{\min }-1} \mathrm{~d} s\right)^{2} \widehat{T_{t} f(x)} .
\end{aligned}
$$

In general, using mathematical induction on $k$, we shall show that

$$
u_{k}(t, x) \leq \frac{\prod_{i=0}^{k-1}\left(n+i\left(\beta_{\max }-1\right)\right)}{k !}\|c\|^{k} p^{k}\left(\int_{0}^{t} \sigma_{s}^{\beta_{\min }-1} \mathrm{~d} s\right)^{k} \widehat{T_{t} f(x)} .
$$

So, suppose that we have shown this for all $k \leq m$. Then, we shall show this for $k=m+1$. Now because of (9), we have

$$
u_{m}(t, x) \leq \frac{\prod_{i=0}^{m-1}\left(n+i\left(\beta_{\max }-1\right)\right)}{m !}\|c\|^{m} p^{m}\left(\int_{0}^{t} \sigma_{s} \beta_{\min }-1 \mathrm{~d} s\right)^{m} \widehat{T_{t} f(x)} .
$$

Now because of (7), we have

$$
\begin{aligned}
u_{m+1}(t, x)= & \int_{0}^{t} \int_{S} \Psi(x, \mathrm{~d} s \mathrm{~d} y) u_{m-1}(t-s, y) \\
\leq & \int_{0}^{t} \sum_{j=1}^{p} c_{i}(x) \int_{S^{n+\beta_{j}-1}} \Psi_{j}(x, \mathrm{~d} s \mathrm{~d} y) u_{k}(t-s, y) \\
\leq & \int_{0}^{t} \mathrm{~d} s\|c\| \sum_{j=1}^{p} \frac{\prod_{i=0}^{m-1}\left(n+\left(\beta_{j}-1\right)+i\left(\beta_{\max }-1\right)\right)}{m !}\|c\|^{m} p^{m} \\
& \times\left(\int_{0}^{t-s} \sigma_{r}^{\beta_{\min }-1} \mathrm{~d} r\right)^{m} \sum_{i=1}^{n} T_{s}\left(c_{j}\left(x_{i}\right) T_{t-s} f\left(x_{i}\right)^{\beta_{j}}\right) \prod_{l=1, l \neq i} T_{s}\left(T_{t-s} f\right)\left(x_{l}\right) \\
\leq & \|c\|^{m+1} p^{m} \sum_{j=1}^{p} \frac{\prod_{i=0}^{m-1}\left(n+\left(\beta_{j}-1\right)+i\left(\beta_{\max }-1\right)\right)}{m !} \\
& \times\left(\int_{0}^{s} \sigma_{r}^{\beta_{\min }-1} \mathrm{~d} r\right)^{m} \sigma_{s}^{\beta_{j}-1} \widehat{T_{t} f(x)}
\end{aligned}
$$




$$
\begin{aligned}
\leq & \|c\|^{m+1} p^{m} \sum_{j=1}^{p} \frac{\prod_{i=0}^{m-1}\left(n+\left(\beta_{j}-1\right)+i\left(\beta_{\max }-1\right)\right)}{m !} \\
& \times\left(\int_{0}^{s} \sigma_{r}^{\beta_{\min }-1} \mathrm{~d} r\right)^{m} \sigma_{s}^{\beta_{\min }-1} \widehat{T_{t} f(x)} \\
\leq & \|c\|^{m+1} p^{m} \sum_{j=1}^{p} \frac{\prod_{i=0}^{m-1}\left(n+\left(\beta_{j}-1\right)+i\left(\beta_{\max }-1\right)\right)}{(m+1) !} \\
& \times\left(\int_{0}^{t} \sigma_{s}^{\beta_{\min }-1} \mathrm{~d} s\right)^{m+1} \widehat{T_{t} f(x)} \\
\leq & \|c\|^{m+1} p^{m+1} \frac{\prod_{i=0}^{m-1}\left(n+(i+1)\left(\beta_{\max }-1\right)\right)}{(m+1) !}\left(\int_{0}^{t} \sigma_{s}^{\beta_{\min }-1} \mathrm{~d} s\right)^{m+1} \widehat{T_{t} f(x)} .
\end{aligned}
$$

Thus, by the induction hypothesis, the proof is complete.

The next result is the analog of Corollary 3.2 of [8].

Corollary 1. Let $u(t, x)$ be the probabilistic solution of (8). Then,

$$
u(t, x) \leq T_{t} f(x)\left(1+\sum_{k=1}^{\infty} v_{k}(t)\right)
$$

where

$$
v_{k}(t)=\frac{\prod_{i=0}^{k-1}\left(1+i\left(\beta_{\max }-1\right)\right)}{k !}\left(\|c\| p \int_{0}^{t} \sigma_{s}^{\beta_{\min }-1} \mathrm{~d} s\right)^{k} .
$$

Hence, we obtain Theorem 2, below, which is similar to Theorem 1.

Theorem 2. For $f \in B^{+}(D)$ satisfying

$$
\left(\beta_{\max }-1\right)\|c\| \int_{0}^{\infty} \sup _{y \in D}\left(T_{t} f(y)\right)^{\beta_{\min }-1} \mathrm{~d} t<1,
$$

there exists a global solution $u(t, x)$ of $(8)$. Moreover, there exists a constant $M>0$ such that

$$
u(t, x) \leq M \widehat{T_{t} f(x)} .
$$

So, we have seen that if there are finitely many terms instead of just one term in the branching part of the semilinear equation, we can solve the problem more easily by closely following the steps of Nagasawa and Sirao. But, if there are infinitely many terms, as in (4), we have to be a little careful. In this case the idea of a common bound for the coefficients in the summation will not help. We need some extra conditions in order to obtain global solutions for our setup. These conditions are given in our main theorem (Theorem 4) in Section 4.

\section{Conditions for the existence of global positive solutions in the unbounded case}

Before examining the conditions for global solutions for the case in which the number of offspring is unbounded, let us study our setup carefully. As mentioned earlier, $A$ is the infinitesimal generator of a linear nonnegative contraction semigroup on the space $B\left(\mathbb{R}^{d}\right)$ of 
bounded measurable functions on $\mathbb{R}^{d}$. Then, we study the existence of global solutions from a probabilistic point of view.

We follow the same approach used by Nagasawa and Sirao [8]. We first prove the following intermediate theorem which gives an upper bound for $u_{k}(t, x)$ for all $k$.

Theorem 3. Let $X$ be an integer-valued random variable taking values $l$ with probability $p_{l}$ for $l=2,3, \ldots$ with $\sum_{i=2}^{\infty} p_{l}=1$. Let $u_{k}(t, x)$ be as defined in Section 2 for each $k$. Then, for $f \in B^{+}\left(\mathbb{R}^{d}\right)$ and $x=\left(x_{1}, x_{2}, \ldots, x_{n}\right) \in\left(\mathbb{R}^{d}\right)^{n}$, we have

$$
u_{k}(t, x) \leq\left(c_{1}(k) \mathrm{E}\left[A_{k}(X) B_{k, t}(X)\right]+c_{2}(k) \mathrm{E}\left[A_{k}(X)\right] \mathrm{E}\left[B_{k, t}(X)\right]\right) \widehat{T_{t} f(x)},
$$

where

$$
\begin{gathered}
A_{k}(X)=\frac{\prod_{i=0}^{k-1}(n+i(X-1))}{k !}, \\
B_{k, t}(X)=\left(\int_{0}^{t} \sigma_{s}^{X-1} \mathrm{~d} s\right)^{k}, \\
\sigma_{s}=\sup _{s} T_{s} f, \\
c_{1}(k)=c_{1}(k-1)+2 c_{2}(k-1),
\end{gathered}
$$

and

$$
c_{2}(k)=2 c_{1}(k-1)+7 c_{2}(k-1)
$$

for $k=1,2,3, \ldots$ with the convention that $c_{1}(1)=1$ and $c_{2}(1)=0$.

Once we prove this theorem, the following corollary is an immediate result.

Corollary 2. Let $u(t, x)$ be the mild solution of (2). Then,

$$
u(t, x) \leq u_{0}(t, x)+\sum_{k=1}^{\infty}\left(c_{1}(k) \mathrm{E}\left[A_{k}(X) B_{k, t}(X)\right]+c_{2}(k) \mathrm{E}\left[A_{k}(X)\right] \mathrm{E}\left[B_{k, t}(X)\right]\right) \widehat{T_{t} f(x)}
$$

Proof. We have

$$
u(t, x)=\sum_{k=0}^{\infty} u_{k}(t, x)=u_{0}(t, x)+\sum_{k=1}^{\infty} u_{k}(t, x) .
$$

Then, we apply the upper bound for $u_{k}(t, x)$ from Theorem 2 to complete the proof.

Next we state our main theorem, which provides the conditions under which we have global solutions to (4).

Theorem 4. Let $X$ be an integer-valued random variable taking the value $l$ with probability $p_{l}$ for $l=2,3, \ldots$ with $\sum_{i=2}^{\infty} p_{l}=1$. Define

$$
\rho(j)=9(j-1) \int_{0}^{\infty} \sigma_{s}^{j-1} \mathrm{~d} s .
$$

Then if $u(t, x)$ is a mild solution of $(4)$, the series in Corollary 2 is convergent if and only if $\rho(j)$ 
is less than 1 for all $j$ ranging from 1 to $\infty$ and inequalities (i) and (ii) below hold, where

(i)

$$
\mathrm{E}\left[\frac{\int_{0}^{\infty} \sigma_{s}^{X-1} \mathrm{~d} s}{1-\rho(X)}\right]<\infty
$$

and

(ii)

$$
\mathrm{E}\left[\frac{(n+X-1)\left(\int_{0}^{\infty} \sigma_{s}^{X-1} \mathrm{~d} s\right)^{2}}{1-\rho(X)}\right]<\infty .
$$

Remark. We can replace (4) in Theorem 4 with the equation

$$
\frac{\partial u}{\partial t}=A u+a \sum_{k=2}^{\infty} p_{k} u^{k}, \quad u(0, x)=f(x), x \in \mathbb{R}^{d},
$$

where $a$ is an arbitrary positive constant. By normalizing, in this case it is sufficient to assume that the $p_{j}$ s are summable.

It is clear that the last two conditions for the existence of global solutions actually give us the following conditions on the $p_{j}$ s:

(i)

$$
\sum_{j=2}^{\infty} \frac{p_{j}}{\left(\int_{0}^{\infty} \sigma_{s}^{j-1} \mathrm{~d} s\right)^{-1}-9(j-1)}<\infty
$$

and

(ii)

$$
\sum_{j=2}^{\infty} \frac{(n+j-1) \int_{0}^{\infty} \sigma_{s}^{j-1} \mathrm{~d} s p_{j}}{\left(\int_{0}^{\infty} \sigma_{s}^{j-1} \mathrm{~d} s\right)^{-1}-9(j-1)}<\infty
$$

So, we shall prove these two conditions while proving Theorem 4 in Section 7.

Note. We can compare conditions (i) and (ii) above with Theorems 1 and 2, which basically give the conditions for global solutions in Nagasawa and Sirao's case and its subsequent intermediate extension. We can see that ' $\rho(j)=9(j-1) \int_{0}^{\infty} \sigma_{s}{ }^{j-1} \mathrm{~d} s<1$ for all $j \geq 2$ ' is basically the condition ' $(\beta-1)\|c\| \int_{0}^{\infty} \sigma_{s}{ }^{\beta-1} \mathrm{~d} s<1$ ' when we have a fixed number of offspring $\beta$ instead of a random number of offspring varying over all positive integers larger than 1 as in our case.

\section{Proof of Theorem 3}

From (7), we observe the following:

$$
\begin{aligned}
u_{1}(t, x) \leq & \int_{0}^{t} \mathrm{E}\left[n \sigma_{s}^{X-1}\right] \mathrm{d} s \widehat{T_{t} f(x)} \\
u_{2}(t, x) \leq & \left(\mathrm{E}\left[\frac{n(n+X-1)}{2}\left(\int_{0}^{t} \sigma_{s}^{X-1} \mathrm{~d} s\right)^{2}\right]\right. \\
& \left.+2 \mathrm{E}\left[\frac{n(n+X-1)}{2}\right] \mathrm{E}\left[\int_{0}^{t} \sigma_{s}^{X-1} \mathrm{~d} s\right]^{2}\right) \widehat{T_{t} f(x)},
\end{aligned}
$$


where $n$ is the number of individuals in the initial population $x=\left(x_{1}, \ldots, x_{n}\right)$. This is obtained as follows. In (6) we have the relation between $\Psi$ and $\Psi_{k}$. Using this, from (7), we have the following:

$$
\begin{aligned}
u_{2}(t, x)= & \int_{0}^{t} \int_{S} \Psi(x, \mathrm{~d} s \mathrm{~d} y) u_{1}(t-s, y) \\
= & \int_{0}^{t} \sum_{k=2}^{\infty} p_{k} \int_{S^{n+k-1}} \Psi_{k}(x, \mathrm{~d} s \mathrm{~d} y) u_{1}(t-s, y) \\
\leq & \int_{0}^{t} \mathrm{~d} s \sum_{k=2}^{\infty} p_{k} \int_{0}^{t-s} \mathrm{E}\left[(n+k-1) \sigma_{r}^{X-1}\right] \mathrm{d} r \\
& \times \sum_{i=1}^{n} T_{S}\left(T_{t-s} f\left(x_{i}\right)^{k}\right) \prod_{l=1, l \neq i} T_{s}\left(T_{t-s} f\right)\left(x_{l}\right) \\
= & \int_{0}^{t} \mathrm{~d} s \sum_{k=2}^{\infty} p_{k}\left(\int_{0}^{s} \mathrm{E}\left[n(n+k-1) \sigma_{r}^{X-1}\right] \mathrm{d} r\right) \sigma_{s}^{k-1} \prod_{l=1}^{n} T_{t} f\left(x_{l}\right) \\
= & \int_{0}^{t} \mathrm{~d} s\left(\int_{0}^{s} \mathrm{E}\left[n(n+Y-1) \sigma_{r}^{X-1} \sigma_{s}^{Y-1}\right] \mathrm{d} r\right) \widehat{T_{t} f(x)},
\end{aligned}
$$

where $Y$ is an integer-valued random variable identically distributed as $X$. Now, in order to simplify this, we use the following. If $X$ and $Y$ are independent and identically distributed random variables then the expectation of a function of these two variables, say $H(X, Y)$, is given by

$$
\begin{aligned}
\mathrm{E}[H(X, Y)]= & \sum_{m=0}^{\infty} \sum_{k=0}^{\infty} H(m, k) \mathrm{P}[X=m, Y=k] \\
= & \sum_{k=0}^{\infty} H(k, k) \mathrm{P}[X=k] \mathrm{P}[Y=k] \\
& +\sum_{k=0}^{\infty} \sum_{m=k+1}^{\infty} H(m, k) \mathrm{P}[X=m] \mathrm{P}[Y=k] \\
& +\sum_{m=0}^{\infty} \sum_{k=m+1}^{\infty} H(m, k) \mathrm{P}[X=m] \mathrm{P}[Y=k] .
\end{aligned}
$$

Using this, $\int_{0}^{t} \mathrm{~d} s\left(\int_{0}^{s} \mathrm{E}\left[n(n+Y-1) \sigma_{r}^{X-1} \sigma_{s}^{Y-1}\right] \mathrm{d} r\right) \widehat{T_{t} f(x)}$ can be written as the sum of three terms. The first term simplifies as follows:

$$
\begin{aligned}
\int_{0}^{t} \mathrm{~d} s \int_{0}^{s} \mathrm{~d} r \sum_{k=2}^{\infty}\left(p_{k}^{2} n(n+k-1) \sigma_{r}^{k-1} \sigma_{s}^{k-1}\right) & \leq \sum_{k=2}^{\infty} p_{k}^{2} n(n+k-1) \int_{0}^{t} \mathrm{~d} s \sigma_{s}^{k-1} \int_{0}^{s} \sigma_{r}^{k-1} \mathrm{~d} r \\
& \leq \sum_{k=2}^{\infty} p_{k}^{2} \frac{n(n+k-1)}{2}\left(\int_{0}^{t} \sigma_{s}^{k-1} \mathrm{~d} s\right)^{2} \\
& \leq \mathrm{E}\left[\frac{n(n+X-1)}{2}\left(\int_{0}^{t} \sigma_{s}^{X-1} \mathrm{~d} s\right)^{2}\right]
\end{aligned}
$$


The second term simplifies as follows:

$$
\begin{array}{rl}
\int_{0}^{t} \mathrm{~d} & s \int_{0}^{s} \mathrm{~d} r \sum_{k=2}^{\infty} \sum_{m=k+1}^{\infty}\left(p_{k} p_{m} n(n+k-1) \sigma_{r}^{m-1} \sigma_{s}^{k-1}\right) \\
& \leq \sum_{k=2}^{\infty} \sum_{m=k+1}^{\infty} p_{k} p_{m} n(n+k-1) \int_{0}^{t} \mathrm{~d} s \sigma_{s}^{k-1} \int_{0}^{s} \sigma_{r}^{k-1} \mathrm{~d} r \\
& \leq \sum_{k=2}^{\infty} \sum_{m=k+1}^{\infty} p_{k} p_{m} \frac{n(n+k-1)}{2}\left(\int_{0}^{t} \sigma_{s}^{k-1} \mathrm{~d} s\right)^{2} \\
& \leq \sum_{k=2}^{\infty} \sum_{m=2}^{\infty} p_{k} p_{m} \frac{n(n+k-1)}{2}\left(\int_{0}^{t} \sigma_{s}^{k-1} \mathrm{~d} s\right)^{2} \\
& \leq \mathrm{E}\left[\frac{n(n+X-1)}{2}\right] \mathrm{E}\left[\left(\int_{0}^{t} \sigma_{s}^{X-1} \mathrm{~d} s\right)^{2}\right] .
\end{array}
$$

The third term simplifies as follows

$$
\begin{aligned}
\int_{0}^{t} \mathrm{~d} s & \int_{0}^{s} \mathrm{~d} r \sum_{m=2}^{\infty} \sum_{k=m+1}^{\infty}\left(p_{k} p_{m} n(n+k-1) \sigma_{r}^{m-1} \sigma_{s}^{k-1}\right) \\
& \leq \sum_{m=2}^{\infty} \sum_{k=m+1}^{\infty} p_{k} p_{m} n(n+k-1) \int_{0}^{t} \mathrm{~d} s \sigma_{s}^{k-1} \int_{0}^{s} \sigma_{r}^{k-1} \mathrm{~d} r \\
& \leq \sum_{m=2}^{\infty} \sum_{k=m+1}^{\infty} p_{k} p_{m} \frac{n(n+k-1)}{2}\left(\int_{0}^{t} \sigma_{s}^{k-1} \mathrm{~d} s\right)^{2} \\
& \leq \sum_{m=2}^{\infty} \sum_{k=2}^{\infty} p_{k} p_{m} \frac{n(n+k-1)}{2}\left(\int_{0}^{t} \sigma_{s}^{k-1} \mathrm{~d} s\right)^{2} \\
& \leq \mathrm{E}\left[\frac{n(n+X-1)}{2}\right] \mathrm{E}\left[\left(\int_{0}^{t} \sigma_{s}^{X-1} \mathrm{~d} s\right)^{2}\right] .
\end{aligned}
$$

Combining all these, we obtain

$$
\begin{aligned}
\int_{0}^{t} \mathrm{~d} s & \left(\int_{0}^{s} \mathrm{E}\left[n(n+Y-1) \sigma_{r}^{X-1} \sigma_{s}^{Y-1}\right] \mathrm{d} r\right) \widehat{T_{t} f(x)} \\
\leq & \mathrm{E}\left[\frac{n(n+X-1)}{2}\left(\int_{0}^{t} \sigma_{s}^{X-1} \mathrm{~d} s\right)^{2}\right] \\
& \left.+2 \mathrm{E}\left[\frac{n(n+X-1)}{2}\right] \mathrm{E}\left[\left(\int_{0}^{t} \sigma_{s}^{X-1} \mathrm{~d} s\right)^{2}\right]\right) \widehat{T_{t} f(x)}
\end{aligned}
$$

Thus, we have obtained (10). Now suppose that we have proved that

$$
u_{k}(t, x) \leq\left(c_{1}(k) \mathrm{E}\left[A_{k}(X) B_{k, t}(X)\right]+c_{2}(k) E\left(A_{k}(X)\right) \mathrm{E}\left[B_{k, t}(X)\right]\right) \widehat{T_{t} f(x)},
$$

where $A_{k}(X), B_{k, t}(X), \sigma_{s}, c_{1}(k)$, and $c_{2}(k)$ are as defined in the statement of Theorem 3 . Now we want to prove the inequality for $u_{k+1}$. In order to do this, first of all, we introduce $D_{k, t}(X, Y)$ 
which is defined as

$$
D_{k, t}(X, Y)=\frac{n \prod_{i=0}^{k-1}(n+(Y-1)+i(X-1))}{k !} \sigma_{t}^{Y-1}=S_{k}(X, Y) \sigma_{t}^{Y-1},
$$

where $S_{k}(X, X)=(k+1)^{-1} A_{k+1}(X)$. Then we use the following lemmas to complete the proof.

Lemma 1. We have

$$
u_{k+1}(t, x) \leq\left(c_{1}(k) P(k, t)+c_{2}(k) Q(k, t)\right) \widehat{T_{t} f(x)},
$$

where $P(k, t)$ and $Q(k, t)$ are given by

$$
\begin{gathered}
P(k, t)=\int_{0}^{t} \mathrm{~d} s \mathrm{E}\left[D_{k, s}(X, Y) B_{k, t-s}(X)\right], \\
Q(k, t)=\int_{0}^{t} \mathrm{~d} s \mathrm{E}\left[D_{k, s}(X, Y)\right] \mathrm{E}\left[B_{k, t-s}(X)\right] .
\end{gathered}
$$

Lemma 2. We have

$$
\begin{aligned}
P(k, t) \leq & \mathrm{E}\left[\frac{\prod_{i=0}^{k}(n+i(X-1))}{(k+1) !}\left(\int_{0}^{t} \sigma_{s}^{X-1} \mathrm{~d} r\right)^{k+1}\right] \\
& +2 \mathrm{E}\left[\frac{\prod_{i=0}^{k}(n+i(X-1))}{(k+1) !}\right] \mathrm{E}\left[\left(\int_{0}^{t} \sigma_{s}^{X-1} \mathrm{~d} r\right)^{k+1}\right] .
\end{aligned}
$$

Lemma 3. We have

$$
\begin{aligned}
Q(k, t) \leq & 2 \mathrm{E}\left[\frac{\prod_{i=0}^{k}(n+i(X-1))}{(k+1) !}\left(\int_{0}^{t} \sigma_{s}^{X-1} \mathrm{~d} r\right)^{k+1}\right] \\
& +7 \mathrm{E}\left[\frac{\prod_{i=0}^{k}(n+i(X-1))}{(k+1) !}\right] \mathrm{E}\left[\left(\int_{0}^{t} \sigma_{s}^{X-1} \mathrm{~d} r\right)^{k+1}\right] .
\end{aligned}
$$

Lemma 4. Using Lemmas 1, 2, and 3, the upper bound for $u_{k+1}(t, x)$ is given by

$$
\left(c_{1}(k+1) \mathrm{E}\left[A_{k+1}(X) B_{k+1, t}(X)\right]+c_{2}(k+1) \mathrm{E}\left[A_{k+1}(X)\right] \mathrm{E}\left[B_{k+1, t}(X)\right]\right) \widehat{T_{t} f(x)},
$$

where

$$
c_{1}(k+1)=c_{1}(k)+2 c_{2}(k)
$$

and

$$
c_{2}(k+1)=2 c_{1}(k)+7 c_{2}(k) .
$$

Once these four lemmas are proved, the proof of Theorem 3 is also complete by the induction hypothesis.

We prove the lemmas in the next section. 


\section{Proof of Lemmas 1-4}

Proof of Lemma 1. To start with, we observe that

$$
\begin{aligned}
u_{k+1}(t, x) & =\int_{0}^{t} \int_{S} \Psi(x, \mathrm{~d} s \mathrm{~d} y) u_{k}(t-s, y) \\
& =\int_{0}^{t} \sum_{l=2}^{\infty} p_{l} \int_{D^{n+k-1}} \Psi^{(l)}(x, \mathrm{~d} s \mathrm{~d} y) u_{k}(t-s, y) \\
& \leq \int_{0}^{t} \sum_{l=2}^{\infty} p_{l} \mathrm{~d} s\left(c_{1}(k) P_{0}(k, t-s)+c_{2}(k) Q_{0}(k, t-s)\right) L_{s, t-s}(x) \\
& =c_{1}(k) M(k, t)+c_{2}(k) N(k, t),
\end{aligned}
$$

where

$$
\begin{gathered}
L_{s, t-s}(x)=\sum_{j=1}^{n} T_{s}\left(h_{t-s}^{l}\right)\left(x_{j}\right) \prod_{j *=1, j * \neq j}^{n} T_{s}\left(h_{t-s}\right)\left(x_{j *}\right) \leq n \sigma_{t-s}^{l-1} \widehat{T_{t} f(x)}, \\
h_{t}(x)=T_{t}(f(x)), \\
P_{0}(k, t-s)=\mathrm{E}\left[\frac{\prod_{i=0}^{k-1}(n+(l-1)+i(X-1))}{k !}\left(\int_{0}^{t-s} \sigma_{r}^{X-1} \mathrm{~d} r\right)^{k}\right], \\
Q_{0}(k, t-s)=\mathrm{E}\left[\frac{\prod_{i=0}^{k-1}(n+(l-1)+i(X-1))}{k !}\right] \mathrm{E}\left[\left(\int_{0}^{t-s} \sigma_{r}^{X-1} \mathrm{~d} r\right)^{k}\right], \\
M(k, t)=\int_{0}^{t} \sum_{l=2}^{\infty} p_{l} \mathrm{~d} s P_{0}(k, t-s) L_{s, t-s}(x), \\
N(k, t)=\int_{0}^{t} \sum_{l=2}^{\infty} p_{l} \mathrm{~d} s Q_{0}(k, t-s) L_{s, t-s}(x) .
\end{gathered}
$$

Therefore,

$$
u_{k+1}(t, x) \leq c_{1}(k) M(k, t)+c_{2}(k) N(k, t) .
$$

Then, we can show that

$$
\begin{aligned}
& M(k, t) \leq P(k, t) \widehat{T_{t} f(x)}, \\
& N(k, t) \leq Q(k, t) \widehat{T_{t} f(x)},
\end{aligned}
$$

where

$$
P(k, t)=\int_{0}^{t} \mathrm{~d} s \mathrm{E}\left[D_{k, s}(X, Y) B_{k, t-s}(X)\right]
$$

and

$$
Q(k, t)=\int_{0}^{t} \mathrm{~d} s \mathrm{E}\left[D_{k, s}(X, Y)\right] \mathrm{E}\left[B_{k, t-s}(X)\right] .
$$

Thus,

$$
u_{k+1}(t, x) \leq\left(c_{1}(k) P(k, t)+c_{2}(k) Q(k, t)\right) \widehat{T_{t} f(x)} .
$$

So, Lemma 1 is proved. 
Proof of Lemma 2. We observe that

$$
\begin{aligned}
P(k, t) & =\int_{0}^{t} \mathrm{~d} s \mathrm{E}\left[D_{k, s}(X, Y) B_{k, t-s}(X)\right] \\
& =\int_{0}^{t} \mathrm{~d} s \sum_{l=2}^{\infty} \sum_{m=2}^{\infty} p_{l} p_{m} D_{k, s}(m, l) B_{k, t-s}(m) \\
& =F(k, s, t-s)+G(k, s, t-s)+H(k, s, t-s),
\end{aligned}
$$

where

$$
\begin{gathered}
F(k, s, t-s)=\int_{0}^{t} \sum_{l=2}^{\infty} p_{l}^{2} S_{k}(l, l)\left(\int_{0}^{t-s} \sigma_{r}^{l-1} \mathrm{~d} r\right)^{k} \sigma_{s}^{l-1} \mathrm{~d} s, \\
G(k, s, t-s)=\int_{0}^{t} \sum_{l=2}^{\infty} \sum_{m=l+1}^{\infty} p_{l} p_{m} S_{k}(l, m)\left(\int_{0}^{t-s} \sigma_{r}^{l-1} \mathrm{~d} r\right)^{k} \sigma_{s}^{m-1} \mathrm{~d} s, \\
H(k, s, t-s)=\int_{0}^{t} \sum_{m=2}^{\infty} \sum_{l=m+1}^{\infty} p_{l} p_{m} S_{k}(l, m)\left(\int_{0}^{t-s} \sigma_{r}^{l-1} \mathrm{~d} r\right)^{k} \sigma_{s}^{m-1} \mathrm{~d} s .
\end{gathered}
$$

Upon simplifying, we obtain the following:

$$
\begin{gathered}
F(k, s, t-s) \leq \mathrm{E}\left[A_{k+1}(X) B_{k+1, t}(X)\right], \\
G(k, s, t-s) \leq \mathrm{E}\left[A_{k+1}(X)\right] \mathrm{E}\left[B_{k+1, t}(X)\right], \\
H(k, s, t-s) \leq \mathrm{E}\left[A_{k+1}(X)\right] \mathrm{E}\left[B_{k+1, t}(X)\right] .
\end{gathered}
$$

Thus, we have

$$
P(k, t) \leq \mathrm{E}\left[A_{k+1}(X) B_{k+1, t}(X)\right]+2 \mathrm{E}\left[A_{k+1}(X)\right] \mathrm{E}\left[B_{k+1, t}(X)\right] .
$$

Hence,

$$
\begin{aligned}
P(k, t) \leq & \mathrm{E}\left[\frac{\prod_{i=0}^{k}(n+i(X-1))}{(k+1) !}\left(\int_{0}^{t} \sigma_{s}^{X-1} \mathrm{~d} s\right)^{k+1}\right] \\
& +2 \mathrm{E}\left[\frac{\prod_{i=0}^{k}(n+i(X-1))}{(k+1) !}\right] \mathrm{E}\left[\left(\int_{0}^{t} \sigma_{s}^{X-1} \mathrm{~d} s\right)^{k+1}\right] .
\end{aligned}
$$

Thus, we have an upper bound for $P(k, t)$.

Proof of Lemma 3. We observe that

$$
\begin{aligned}
Q(k, t) & =\int_{0}^{t} \mathrm{~d} s \mathrm{E}\left[D_{k, s}(X, Y)\right] \mathrm{E}\left[B_{k, t-s}(X)\right] \\
& =\int \mathrm{d} s \sum_{l=2}^{\infty} \sum_{m=2}^{\infty} \sum_{j=2}^{\infty} p_{l} p_{m} p_{j} D_{k, s}(m, l) B_{k, t-s}(j) \\
& =U(k, s, t-s)+V(k, s, t-s)+W(k, s, t-s),
\end{aligned}
$$


where

$$
\begin{gathered}
U(k, s, t-s)=\int \sum_{l=2}^{\infty} \sum_{m=2}^{\infty} p_{l} p_{m}^{2} S_{k}(l, m) \sigma_{s}^{m-1}\left(\int_{0}^{t-s} \sigma_{r}^{m-1} \mathrm{~d} r\right)^{k} \mathrm{~d} s, \\
V(k, s, t-s)=\int \sum_{l=2}^{\infty} \sum_{m=2}^{\infty} \sum_{j=m+1}^{\infty} p_{l} p_{m} p_{j} S_{k}(l, m) \sigma_{s}^{m-1}\left(\int_{0}^{t-s} \sigma_{r}^{j-1} \mathrm{~d} r\right)^{k} \mathrm{~d} s, \\
W(k, s, t-s)=\int \sum_{l=2}^{\infty} \sum_{j=2}^{\infty} \sum_{m=j+1}^{\infty} p_{l} p_{m} p_{j} S_{k}(l, m) \sigma_{s}^{m-1}\left(\int_{0}^{t-s} \sigma_{r}^{j-1} \mathrm{~d} r\right)^{k} \mathrm{~d} s .
\end{gathered}
$$

Now, we can show that both $U(k, s, t-s)$ and $V(k, s, t-s)$ are less that $Z(k, s, t-s)$, where

$$
Z(k, s, t-s)=\sum_{l=2}^{\infty} \sum_{m=2}^{\infty} p_{l} p_{m}(k+1)^{-1} S_{k}(l, m)\left(\int_{0}^{t} \sigma_{s}^{m-1} \mathrm{~d} s\right)^{k+1} .
$$

We can show that

$$
Z(k, s, t-s) \leq \mathrm{E}\left[A_{k+1}(X) B_{k+1, t}(X)\right]+2 \mathrm{E}\left[A_{k+1}(X)\right] \mathrm{E}\left[B_{k+1, t}(X)\right] .
$$

Also,

$$
W(k, s, t-s)=\mathrm{E}\left[(k+1)^{-1} S_{k}(X, Y)\right] \mathrm{E}\left[\int_{0}^{t} \sigma_{s}^{X-1} \mathrm{~d} s\right]^{k+1} .
$$

But,

$$
\mathrm{E}\left[(k+1)^{-1} S_{k}(X, Y)\right] \leq 3 \mathrm{E}\left[A_{k+1}(X)\right] .
$$

Therefore,

$$
W(k, s, t-s) \leq 3 \mathrm{E}\left[A_{k+1}(X)\right] \mathrm{E}\left[B_{k+1, t}(X)\right] .
$$

Thus, we have

$$
\begin{aligned}
Q(k, t)= & U(k, s, t-s)+V(k, s, t-s)+W(k, s, t-s) \\
\leq & 2 Z(k, s, t-s)+W(k, s, t-s) \\
\leq & 2\left(\mathrm{E}\left[A_{k+1}(X) B_{k+1, t}(X)\right]+2 \mathrm{E}\left[A_{k+1}(X)\right] \mathrm{E}\left[B_{k+1, t}(X)\right]\right) \\
& +3 \mathrm{E}\left[A_{k+1}(X)\right] \mathrm{E}\left[B_{k+1, t}(X)\right] \\
= & 2 \mathrm{E}\left[A_{k+1}(X) B_{k+1, t}(X)\right]+7 \mathrm{E}\left[A_{k+1}(X)\right] \mathrm{E}\left[B_{k+1, t}(X)\right] .
\end{aligned}
$$

Hence,

$$
\begin{aligned}
Q(k, t) \leq & 2 \mathrm{E}\left[\frac{\prod_{i=0}^{k}(n+i(X-1))}{(k+1) !}\left(\int_{0}^{t} \sigma_{s}^{X-1} \mathrm{~d} s\right)^{k+1}\right] \\
& +7 \mathrm{E}\left[\frac{\prod_{i=0}^{k}(n+i(X-1))}{(k+1) !}\right] \mathrm{E}\left[\left(\int_{0}^{t} \sigma_{s}^{X-1} \mathrm{~d} s\right)^{k+1}\right] .
\end{aligned}
$$

Thus, we have an upper bound for $Q(k, t)$ also. 
Proof of Lemma 4. Combining the upper bounds for $P(k, t)$ and $Q(k, t)$ from Lemma 2 and Lemma 3, we ultimately obtain an upper bound for $u_{k+1}(t, x)$ :

$$
\begin{aligned}
\left(c_{1}(k) P\right. & \left.(k, t)+c_{2}(k) Q(k, t)\right) \widehat{T_{t} f(x)} \\
\leq & \left(\left(c_{1}(k)+2 c_{2}(k)\right) \mathrm{E}\left[A_{k+1}(X) B_{k+1, t}(X)\right]\right. \\
& \left.+\left(2 c_{1}(k)+7 c_{2}(k)\right) \mathrm{E}\left[A_{k+1}(X)\right] \mathrm{E}\left[B_{k+1, t}(X)\right]\right) \widehat{T_{t} f(x)} \\
\leq & \left(c_{1}(k+1) \mathrm{E}\left[A_{k+1}(X) B_{k+1, t}(X)\right]+c_{2}(k+1) \mathrm{E}\left[A_{k+1}(X)\right] \mathrm{E}\left[B_{k+1, t}(X)\right]\right) \widehat{T_{t} f(x)} .
\end{aligned}
$$

Thus,

$u_{k+1}(t, x) \leq\left(c_{1}(k+1) \mathrm{E}\left[A_{k+1}(X) B_{k+1, t}(X)\right]+c_{2}(k+1) \mathrm{E}\left[A_{k+1}(X)\right] \mathrm{E}\left[B_{k+1, t}(X)\right]\right) \widehat{T_{t} f(x)}$,

where $c_{1}(k+1)=c_{1}(k)+2 c_{2}(k)$ and $c_{2}(k+1)=2 c_{1}(k)+7 c_{2}(k)$. Here,

$$
A_{k+1}(X)=\frac{\prod_{i=0}^{k}(n+i(X-1))}{(k+1) !} \quad \text { and } \quad B_{k+1, t}(X)=\left(\int_{0}^{t} \sigma_{s}^{X-1} \mathrm{~d} s\right)^{k+1} .
$$

Thus, the bounds are correct for all $k \geq 1$. For $k=1, c_{1}(1)=1$ and $c_{2}(1)=0$.

\section{Proof of Theorem 4: conditions for global solutions}

It was shown in Corollary 2 that if $u(t, x)$ is a mild solution then

$$
u(t, x) \leq u_{0}(t, x)+\sum_{k=1}^{\infty}\left(c_{1}(k) \mathrm{E}\left[A_{k}(X) B_{k, t}(X)\right]+c_{2}(k) \mathrm{E}\left[A_{k}(X)\right] \mathrm{E}\left[B_{k, t}(X)\right]\right) \widehat{T_{t} f(x)} .
$$

We are interested in the series appearing on the right-hand side of the above inequality. If this series is convergent then it is clear that we have global solutions to (4). So, we show that this series is convergent. We can observe that the given series is actually a sum of two series, namely

$$
\sum_{k=1}^{\infty} c_{1}(k) \mathrm{E}\left[A_{k}(X) B_{k, t}(X)\right]
$$

and

$$
\sum_{k=2}^{\infty} c_{2}(k) \mathrm{E}\left[A_{k}(X)\right] \mathrm{E}\left[B_{k, t}(X)\right]
$$

Firstly, we shall look at the former series. Now,

$$
\sum_{k=1}^{\infty} c_{1}(k) \mathrm{E}\left[A_{k}(X) B_{k, t}(X)\right]=\sum_{k=1}^{\infty} c_{1}(k) \sum_{j=2}^{\infty} p_{j} A_{k}(j) B_{k, t}(j) .
$$

We shall verify that, under the assumptions in Theorem 4, the series

$$
\sum_{k=1}^{\infty} c_{1}(k) \sum_{j=2}^{\infty} p_{j} A_{k}(j) B_{k}(j)
$$


is convergent, where

$$
B_{k, t}(j)=\left(\int_{0}^{t} \sigma_{s}^{j-1} \mathrm{~d} s\right)^{k} \leq B_{k}(j)=\left(\int_{0}^{\infty} \sigma_{s}^{j-1} \mathrm{~d} s\right)^{k} \text { for all } t .
$$

Now,

$$
\sum_{k=1}^{\infty} c_{1}(k) \sum_{j=2}^{\infty} p_{j} A_{k}(j) B_{k}(j)=\sum_{j=2}^{\infty} p_{j} \sum_{k=1}^{\infty} c_{1}(k) A_{k}(j) B_{k}(j) .
$$

So, let us first obtain conditions for the convergence of the inner series on the right-hand side. By the ratio test, the inner series is convergent if

$$
\begin{gathered}
\frac{c_{1}(k+1)}{c_{1}(k)} \frac{A_{k+1}(j)}{A_{k}(j)} \frac{B_{k+1}(j)}{B_{k}(j)}<1, \\
\frac{c_{1}(k+1)}{c_{1}(k)}(j-1) \int_{0}^{\infty} \sigma_{s}^{j-1} \mathrm{~d} s<1,
\end{gathered}
$$

or

$$
9(j-1) \int_{0}^{\infty} \sigma_{s}^{j-1} \mathrm{~d} s<1,
$$

since $c_{1}(k+1) / c_{1}(k) \leq 9$.

Now, $9(j-1) \int_{0}^{\infty} \bar{\sigma}_{s}^{j-1} \mathrm{~d} s=\rho(j)<1$ for all $j$ by hypothesis. Then,

$$
\sum_{k=1}^{\infty} c_{1}(k) A_{k}(j) B_{k}(j)<\infty .
$$

Let $c_{1}(k) A_{k}(j) B_{k}(j)=a_{k, j}$ for all $j$. As a consequence,

$$
\sum_{k=1}^{\infty} a_{k, j}<\sum_{k=1}^{\infty} \rho(j)^{k-1} a_{1, j}=\frac{a_{1, j}}{1-\rho(j)} .
$$

Then we have

$$
\begin{aligned}
\sum_{j=2}^{\infty} p_{j} \sum_{k=1}^{\infty} a_{k, j} & <\sum_{j=2}^{\infty} p_{j} \frac{a_{1, j}}{1-\rho(j)} \\
& =\sum_{j=2}^{\infty} p_{j} \frac{c_{1}(1) A_{1}(j) B_{1}(j)}{1-\rho(j)} \\
& =n \sum_{j=2}^{\infty} \frac{p_{j}}{\left(\int_{0}^{\infty} \sigma_{s}^{j-1} \mathrm{~d} s\right)^{-1}-9(j-1)},
\end{aligned}
$$

which is less than $\infty$ by Theorem 4(i). So, we have verified that the first series is convergent under the given hypothesis.

Now we shall look at the latter series, $\sum_{k=2}^{\infty} c_{2}(k) \mathrm{E}\left[A_{k}(X)\right] \mathrm{E}\left[B_{k, t}(X)\right]$. As before, we shall verify that

$$
\sum_{k=2}^{\infty} c_{2}(k) \mathrm{E}\left[A_{k}(X)\right] \mathrm{E}\left[B_{k}(X)\right]<\infty
$$


where $B_{k}(X)=\left(\int_{0}^{\infty} \sigma_{s}^{X-1} \mathrm{~d} s\right)^{k}$. Now,

$$
\begin{aligned}
\sum_{k=2}^{\infty} c_{2}(k) \mathrm{E}\left[A_{k}(X)\right] \mathrm{E}\left[B_{k}(X)\right] & \\
= & \sum_{k=2}^{\infty} c_{2}(k) \sum_{j_{1}=2}^{\infty} \sum_{j_{2}=2}^{\infty} p_{j_{1}} p_{j_{2}} A_{k}\left(j_{1}\right) B_{k}\left(j_{2}\right) \\
& =\sum_{j_{1}=2}^{\infty} \sum_{j_{2}=2}^{\infty} p_{j_{1}} p_{j_{2}} \sum_{k=2}^{\infty} c_{2}(k) A_{k}\left(j_{1}\right) B_{k}\left(j_{2}\right) .
\end{aligned}
$$

We write this as the sum of three terms, namely

$$
\begin{gathered}
\sum_{j_{1}=2}^{\infty} p_{j_{1}}^{2} \sum_{k=2}^{\infty} c_{2}(k) A_{k}\left(j_{1}\right) B_{k}\left(j_{1}\right), \\
\sum_{j_{2}=2}^{\infty} \sum_{j_{1}=j_{2}+1}^{\infty} p_{j_{1}} p_{j_{2}} \sum_{k=2}^{\infty} c_{2}(k) A_{k}\left(j_{2}\right) B_{k}\left(j_{1}\right),
\end{gathered}
$$

and

$$
\sum_{j_{1}=2}^{\infty} \sum_{j_{2}=j_{1}+1}^{\infty} p_{j_{1}} p_{j_{2}} \sum_{k=2}^{\infty} c_{2}(k) A_{k}\left(j_{1}\right) B_{k}\left(j_{2}\right)
$$

Now the first term satisfies

$$
\sum_{j_{1}=2}^{\infty} p_{j_{1}}^{2} \sum_{k=2}^{\infty} c_{2}(k) A_{k}\left(j_{1}\right) B_{k}\left(j_{1}\right) \leq \sum_{j_{1}=2}^{\infty} p_{j_{1}} \sum_{k=2}^{\infty} c_{2}(k) A_{k}\left(j_{1}\right) B_{k}\left(j_{1}\right) .
$$

The second term satisfies

$$
\sum_{j_{2}=2}^{\infty} \sum_{j_{1}=j_{2}+1}^{\infty} p_{j_{1}} p_{j_{2}} \sum_{k=2}^{\infty} c_{2}(k) A_{k}\left(j_{2}\right) B_{k}\left(j_{2}\right) \leq \sum_{j_{2}=2}^{\infty} p_{j_{2}} \sum_{k=2}^{\infty} c_{2}(k) A_{k}\left(j_{2}\right) B_{k}\left(j_{2}\right) .
$$

And the third term satisfies

$$
\sum_{j_{1}=2}^{\infty} \sum_{j_{2}=j_{1}+1}^{\infty} p_{j_{1}} p_{j_{2}} \sum_{k=2}^{\infty} c_{2}(k) A_{k}\left(j_{1}\right) B_{k}\left(j_{2}\right) \leq \sum_{j_{1}=2}^{\infty} p_{j_{1}} \sum_{k=2}^{\infty} c_{2}(k) A_{k}\left(j_{1}\right) B_{k}\left(j_{1}\right) .
$$

Thus, from (11), (12), and (13), we can conclude that the second series satisfies

$$
\sum_{k=2}^{\infty} c_{2}(k) \mathrm{E}\left[A_{k}(X)\right] \mathrm{E}\left[B_{k}(X)\right] \leq 3 \sum_{j=2}^{\infty} p_{j} \sum_{k=2}^{\infty} c_{2}(k) A_{k}(j) B_{k}(j) .
$$

Now, we can argue exactly as in the case of the first series and conclude that the right-hand side satisfies

$$
3 \sum_{j=2}^{\infty} p_{j} \sum_{k=2}^{\infty} c_{2}(k) A_{k}(j) B_{k}(j) \leq 3 \sum_{j=2}^{\infty} p_{j} \frac{c_{2}(2) A_{2}(j) B_{2}(j)}{1-\rho(j)} .
$$


The right-hand side of the above inequality can be simplified to arrive at condition (ii) in the hypothesis, i.e.

$$
\sum_{j=2}^{\infty} \frac{(n+j-1) \int_{0}^{\infty} \sigma_{s}^{j-1} \mathrm{~d} s p_{j}}{\left(\int_{0}^{\infty} \sigma_{s}^{j-1} \mathrm{~d} s\right)^{-1}-9(j-1)}<\infty .
$$

Thus, the second series is also finite by condition (ii) of Theorem 4 . Hence, since both series are finite under the given conditions, the proof of Theorem 4 is complete. So, we conclude that the conditions given in Theorem 4 are indeed the required conditions for global solutions in our case.

\section{Some interesting examples}

Here we apply Theorem 4 to some interesting cases.

The first example that we consider is the following:

$$
\frac{\partial u}{\partial t}=A u(t, x)+\sum_{k=2}^{\infty} p_{k} u^{k}(t, x), \quad u(0, x)=f(x),
$$

where we are assuming that $A$ is such that $\sigma_{s}=\sup _{s} T_{s} f=\mathrm{e}^{-10 s}$ and that the $p_{j}$ s are of the order of $(j-1)^{-2}$. Then, we go back to the conditions of Theorem 4. Condition (i) of Theorem 4 leads us to

$$
\sum_{j=2}^{\infty} \frac{p_{j}}{\left(\int_{0}^{\infty} \sigma_{s}^{j-1} \mathrm{~d} s\right)^{-1}-9(j-1)}=\sum_{j=2}^{\infty} \frac{p_{j}}{(j-1)} .
$$

Now this is finite because of the assumption that the $p_{j}$ s are of the order of $(j-1)^{-2}$. To verify condition (ii) of Theorem 4 , we proceed as follows. We have to show that

$$
\sum_{j=2}^{\infty} \frac{(n+j-1) \int_{0}^{\infty} \sigma_{s}^{j-1} \mathrm{~d} s p_{j}}{\left(\int_{0}^{\infty} \sigma_{s}^{j-1} \mathrm{~d} s\right)^{-1}-9(j-1)}<\infty
$$

This is now equal to

$$
\sum_{j=2}^{\infty} \frac{p_{j}}{(j-1)} \frac{n+j-1}{10(j-1)}=\frac{n}{10} \sum_{j=2}^{\infty} \frac{p_{j}}{(j-1)^{2}}+\sum_{j=2}^{\infty} \frac{p_{j}}{10(j-1)} .
$$

The first term on the right-hand side is always finite and the second term on the right-hand side is finite because of the assumption that the $p_{j}$ s are of the order of $(j-1)^{-2}$.

In the second example we consider symmetric $\alpha$-stable processes. Let $T_{t}$ be a $d$-dimensional symmetric $\alpha$-stable process. Then, it is known that

$$
T_{t} f(x)=\int_{R^{d}} p(t, x-y) f(y) \mathrm{d} y
$$

and

$$
\exp \left(-t|z|^{\alpha}\right)=\int_{R^{d}} \mathrm{e}^{\mathrm{i}(z, x)} p(t, x) \mathrm{d} x .
$$

Then Theorem 3.5 of [8] gives us a condition on $f$ under which their semilinear equation had a global solution provided that $d(\beta-1) / \alpha>1$, where $\beta$ was the fixed number of offspring generated by each parent. We shall now see that a similar condition will give rise to global solutions in our setup as well. 
Theorem 5. Let $T_{t}$ be a d-dimensional symmetric $\alpha$-stable process, where $d>4$. Let $\eta$ be a positive number. Also, assume that our initial data satisfies

$$
0 \leq f(x) \leq \delta p(t+\eta, x)
$$

for a sufficiently small positive number $\delta$. Then there exists a global solution $u(t, x)$ to our semilinear equation (4) where $a(x)$ is identically equal to 1.

Proof. Since $d \geq 4$, we know that $d / \alpha$ is bigger than 2 . Then $d(j-1) / \alpha$ is bigger than 2 for all $j \geq 2$. Also, by hypothesis, our initial data $f$ satisfies

$$
0 \leq f(x) \leq \delta p(t+\eta, x)
$$

for some positive numbers $\delta$ and $\eta$. Then, following the steps in Theorem 3.5 of [8], we arrive at

$$
\int_{0}^{\infty} \sigma_{s}^{j-1} \mathrm{~d} s \leq \delta^{j-1} p(1,0)^{j-1} \frac{\eta^{1-d(j-1) / \alpha}}{d(j-1) / \alpha-1} .
$$

Now, for the existence of global solutions, we need to verify the conditions of Theorem 4 , namely

(i)

$$
\sum_{j=2}^{\infty} \frac{p_{j}}{\left(\int_{0}^{\infty} \sigma_{s}^{j-1} \mathrm{~d} s\right)^{-1}-9(j-1)}<\infty
$$

and

(ii)

$$
\sum_{j=2}^{\infty} \frac{(n+j-1) \int_{0}^{\infty} \sigma_{s}^{j-1} \mathrm{~d} s p_{j}}{\left(\int_{0}^{\infty} \sigma_{s}^{j-1} \mathrm{~d} s\right)^{-1}-9(j-1)}<\infty
$$

So, from (15), we have

$$
\left(\int_{0}^{\infty} \sigma_{s}^{j-1} \mathrm{~d} s\right)^{-1}-9(j-1) \geq \frac{d(j-1) / \alpha-1}{\delta^{j-1} p(1,0)^{j-1} \eta^{1-d(j-1) / \alpha}}-9(j-1) .
$$

This implies that

$$
\left(\int_{0}^{\infty} \sigma_{s}^{j-1} \mathrm{~d} s\right)^{-1}-9(j-1) \geq \frac{d(j-1) / \alpha-1-9(j-1) \delta^{j-1} p(1,0)^{j-1} \eta^{1-d(j-1) / \alpha}}{\delta^{j-1} p(1,0)^{j-1} \eta^{1-d(j-1) / \alpha}},
$$

which then implies that

$$
\begin{aligned}
& \left(\left(\int_{0}^{\infty} \sigma_{s}^{j-1} \mathrm{~d} s\right)^{-1}-9(j-1)\right)^{-1} \\
& \quad \leq \frac{\delta^{j-1} p(1,0)^{j-1} \eta^{1-d(j-1) / \alpha}}{d(j-1) / \alpha-1-9(j-1) \delta^{j-1} p(1,0)^{j-1} \eta^{1-d(j-1) / \alpha}}
\end{aligned}
$$

Now, since $\eta^{1-d(j-1) / \alpha} \leq 1$, the numerator on the right-hand side of (16) is less than or equal to $\delta^{j-1} p(1,0)^{j-1}$. Similarly, the denominator on the right-hand side of (16) is greater 
than or equal to $d(j-1) / \alpha-1-9(j-1) \delta^{j-1} p(1,0)^{j-1}$. Now, we can choose $\delta$ so small that, for large $j$ (say $j>J$ ), we have

$$
\delta^{j-1} p(1,0)^{j-1}<M^{-(j-1)}<(j-1)^{-2}
$$

for some positive number $M$. Then (16) becomes

$$
\left(\left(\int_{0}^{\infty} \sigma_{s}^{j-1} \mathrm{~d} s\right)^{-1}-9(j-1)\right)^{-1} \leq \frac{(j-1)^{-2}}{d(j-1) / \alpha-1-9(j-1)^{-1}}
$$

Now, for large $j$, we have $9(j-1)^{-1} \leq 1$, so the denominator is greater than or equal to $d(j-1) / \alpha-2$. As a result, the right-hand side of the above inequality is of the order of $(j-1)^{-3}$. So, for some large $J^{*}>J$, we have

$$
\sum_{j=J^{*}}^{\infty} \frac{p_{j}}{\left(\int_{0}^{\infty} \sigma_{s}^{j-1} \mathrm{~d} s\right)^{-1}-9(j-1)} \leq \sum_{j=J^{*}}^{\infty} \frac{p_{j}}{d(j-1)^{3} / \alpha-2(j-1)^{2}},
$$

which is less than $\infty$, and, hence,

$$
\sum_{j=0}^{\infty} \frac{p_{j}}{\left(\int_{0}^{\infty} \sigma_{s}^{j-1} \mathrm{~d} s\right)^{-1}-9(j-1)}<\infty
$$

Now, because of the observation made while verifying Theorem 4(i) we have, for large $j$,

$$
\left(\left(\int_{0}^{\infty} \sigma_{s}^{j-1} \mathrm{~d} s\right)^{-1}-9(j-1)\right)^{-1} \leq \frac{(j-1)^{-2}}{d(j-1) / \alpha-1-9(j-1)^{-1}}
$$

Now, because of the discussion above with respect to verifying Theorem 4(i), from (15), we have

$$
\int_{0}^{\infty} \sigma_{s}^{j-1} \mathrm{~d} s \leq \frac{(j-1)^{-2}}{d(j-1) / \alpha-1} .
$$

Therefore, from the second condition, for some large $j=J^{* *}$, we have

$$
\sum_{j=J^{* *}}^{\infty} \frac{(n+j-1) \int_{0}^{\infty} \sigma_{s}^{j-1} \mathrm{~d} s p_{j}}{\left(\int_{0}^{\infty} \sigma_{s}^{j-1} \mathrm{~d} s\right)^{-1}-9(j-1)} \leq \sum_{j=J^{* *}}^{\infty} \frac{(n+j-1) p_{j}}{(j-1)^{4}(d(j-1) / \alpha-1)(d(j-1) / \alpha-2)},
$$

which is obviously less than $\infty$ and, hence, the whole series is less than $\infty$. Therefore, using similar arguments as in the case of Theorem 4(i), we can conclude that Theorem 4(ii) is also satisfied.

Hence, we have seen that the conditions for the existence of global solutions are satisfied for symmetric $\alpha$-stable processes.

Remark. The assumptions for the existence of global solutions for symmetric $\alpha$-stable processes in the case of a fixed number of offspring for each individual (Nagasawa and Sirao's case) are exactly the same as the case of a random number of offspring for an individual parent (our case). This follows by comparing Theorem 3.5 of [8] and our Theorem 5 . 


\section{References}

[1] Fujita, H. (1966). On the blowing up of solutions of the Cauchy problem for $u_{t}=\Delta u+u^{1=\alpha}$ J. Fac. Sci. Univ. Tokyo 13, 109-124.

[2] Ikeda, N., Nagasawa, M. And Watanabe, S. (1965). Branching Markov processes. Abstracts Proc. Japan Acad. 41, 816-821.

[3] Ikeda, N., Nagasawa, M. and Watanabe, S. (1966). Branching Markov processes. Abstracts Proc. Japan Acad. 42, 252-257, 370-375, 380-384, 719-724, 1016-1021, 1022-1026.

[4] Ikeda, N., Nagasawa, M. and Watanabe, S. (1968). Branching Markov processes. I. J. Math. Kyoto Univ. 8, 233-278.

[5] Ikeda, N., Nagasawa, M. and Watanabe, S. (1968). Branching Markov processes. II. J. Math. Kyoto Univ. $\mathbf{8}, 365-410$.

[6] López-Mimbela, J. A. (1996). A probabilistic approach to existence of global solutions of a system of nonlinear differential equations. Aportaciones Matematicas Serie Notas de Investigacion 12, 147-155.

[7] López-Mimbela, J. A. And Wakolbinger, A. (1998). Length of Galton-Watson trees and blow-up of semilinear systems. J. Appl. Prob. 35, 802-811.

[8] Nagasawa, M. and Sirao, T. (1969). Probabilistic treatment of the blowing up of solutions for a nonlinear integral equation. Trans Amer. Math. Soc. 139, 301-310. 